\title{
SERES INVISÍVEIS: UM PANORAMA SOBRE A CONDIÇÃO DE PORTADORES DE DEFICIÊNCIA NO JAPÃO ${ }^{1}$ \\ INVISIBLE BEINGS: AN OVERVIEW OF PEOPLE WITH DISABILITIES' CIRCUMSTANCES IN JAPAN
}

\author{
Beatriz Kaori Miyakoshi Lopes ${ }^{2}$ \\ Alexandre Ratsuo Uehara ${ }^{3}$
}

Resumo: Apesar de o Japão ter ratificado a Convenção sobre Direitos de Pessoas com Deficiências em 2014 e Tóquio ser a primeira cidade no mundo que sediará os Jogos Paralímpicos mais de uma vez, portadores de deficiência são geralmente segregados da sociedade e considerados invisíveis. Este processo de separação se inicia na infância e perdura durante suas vidas. Este trabalho objetiva traçar um panorama sobre suas condições de vida, principais leis e eventos recentes.

Palavras-chave: Deficiência; Japão; Direitos humanos; Minorias; Paralimpíadas.

Abstract: Despite the fact that Japan ratified the Convention on the Rights of Persons with Disabilities in 2014 and that Tokyo will be the first city in the world to host the Paralympic Games more than once, people with disabilities are usually segregated from society and regarded as invisible. This separation process starts at childhood and lingers throughout their lives. This work aims to outline an overview of their living conditions, the main laws and recent events.

Keywords: Disability; Japan; Human rights; Minorities; Paralympics.

\section{INTRODUÇÃO}

Com os Jogos Paralímpicos a caminho para 2021 em Tóquio, as condições de vida de portadores de deficiência estão cada vez mais em foco, tanto na academia quanto na mídia. O doutor Etienne Krug, um dos diretores da Organização Mundial da Saúde

1 Artigo submetido em 11/06/2020 e aceito em 25/08/2020.

2 Mestranda no Programa de Língua, Literatura e Cultura Japonesa da Universidade de São Paulo; biakaori@usp.br (ORCID iD: https://orcid.org/0000-0002-0701-4187).

3 Professor da Escola Superior de Propaganda e Marketing; Doutor em Ciências Políticas pela Universidade de São Paulo; aruehara@usp.br; ORCID iD: https://orcid.org/0000-0002-9262-4472). 
(OMS), descreve o evento: "As Paralimpíadas são um dos eventos mundiais mais proeminentes nos quais pessoas com deficiencias demostram seu tremendo talento e energia (...)", contudo, ele observa que "demasiados portadores não desfrutam nem do direito humano mais básico" (KRUG, 2004).

Os direitos referidos por Krug seriam, por exemplo, os de livre locomoção (em decorrência de infraestrutura inadequada), acesso à educação (escolas convencionais não possuem estrutura para alunos com deficiência, escolas especiais não existem ou são financeiramente inacessíveis) e de identidade (vítimas de massacres não serem identificadas). Vários deficientes são, contudo, privados desses direitos.

Este artigo explanará suas condições no Japão, país este que passa por um processo de transformação social, com o aumento da visibilidade e de direitos de portadores. Contudo, recentes eventos, como a tragédia em Sagamihara e a Lei Eugênica, demonstram que ainda há um longo caminho a ser percorrido, principalmente no quesito da segregação de deficientes. Há uma tradição de escondê-los por parte de suas famílias e, mesmo durante sua criação e ao começar a trabalhar, eles vivem separadamente da sociedade japonesa.

As Paralimpíadas serão um novo estágio neste processo de transformação, pois proporcionarão uma oportunidade para mostrar pessoas reais com vários tipos de deficiência sendo excelentes em seus respectivos esportes para bilhões de pessoas no mundo. O Japão já tem história com as Paralimpíadas: o país começou a participar em 1964, quando foi anfitrião nas Olímpicas de verão e depois também dos Jogos Paralímpicos de Inverno em 1972 (Sapporo) e em 1998 (Nagano).

\section{CONDIÇÃO DE DEFICIÊNCIA}

Portadores de deficiência contabilizam $15 \%$ da população mundial, o equivalente a aproximadamente um bilhão de pessoas. Esta é uma minoria populacional diferenciada devido a dois fatores: 1) por ser a maior minoria populacional existente (MAIOR, 2016, p.3) e por 2) possuir a diversidade como uma de suas características mais evidentes, diferentemente de outros grupos sociais que são visivelmente homogêneos e possuem necessidades compartilhadas (CARVALHO, 2012).

$\mathrm{O}$ artigo 2 da Lei Básica para Pessoas com Deficiências, primeiro documento japonês referente a esse grupo, de 1970, define pessoas com deficiência como "indivíduos que têm suas vivências diárias e sociais substancialmente e continuamente limitadas devido a deficiências física, intelectual ou mental" (CABINET OFFICE, 2011, p.1).

No passado, os deficientes foram vistos como indivíduos falhos, com condições temíveis e sem expectativa de cumprir com as expectativas sociais, como trabalhar ou ter filhos. Os crescentes movimentos de direitos humanos, vida autônoma e autodeterminação subverteram este modelo e introduziram novos valores, como a identidade positiva, a dignidade e o orgulho (HEYER, 2000a). 
Os portadores de deficiência passaram a ser vistos como membros ativos da sociedade, sendo capazes de exercitar seus próprios direitos (LO, 2012). A deficiência é vista como um problema encontrado "dentro da sociedade, em vez de estar nos indivíduos que, por acaso, possuem impedimentos" (FRENCH, 1993, p.16, apud STIBBE, 2004, p.22). A maneira encontrada para reduzir tal problema é ajustando o ambiente social e físico para garantir que deficientes tenham suas necessidades e direitos atendidos.

Os principais tipos de barreiras enfrentadas por deficientes são: (i) suas necessidades não serem consideradas em políticas públicas; (ii) preconceitos e equívocos que dificultam a participação social; (iii) insuficiência de serviços principalmente nas áreas de saúde e reabilitação; (iv) coordenação ineficiente de serviços; (v) investimento inadequado; (vi) falta de acessibilidade, principalmente em transporte e comunicação (WORLD HEALTH ORGANIZATION - WHO, 2011).

$\mathrm{O}$ tema de direitos dos deficientes só passou a ter âmbito internacional graças à atuação da Organização das Nações Unidas (ONU), em 1981, com a inauguração do Ano Internacional de Deficientes (HEYER, 2000b). Mas a principal mudança ocorreu em 2006, com a Convenção sobre Direitos de Pessoas com Deficiências, o primeiro tratado multilateral abrangente no tema de deficiências, marcando uma mudança de paradigma. Seus signatários se comprometem a proibir a discriminação, providenciar serviços de reabilitação e de treino vocacional, promover empregos tanto no setor público quanto no privado, além de garantir uma "acomodação razoável" no local de trabalho (ONU, 2006, apud LO, 2012).

O Japão assinou a Convenção em 2007, mas só a ratificou em 2014, tornando-se o $140^{\circ}$ país a fazê-lo, depois da China e Coreia do Sul (SHIRASAWA, 2014). O governo japonês não havia apresentado políticas suficientemente efetivas de acordo com a Convenção e sua ratificação foi bloqueada pelo Fórum de Deficiências nacional. Em 2010, foi criado um Comitê para a Reforma Política de Deficiências que implementou uma série de reformas, como a emenda em 2014 para a lei de 1970 e a criação do Ato de Eliminação de Discriminação de Pessoas com Deficiência em 2013 (NAGASE, 2013).

Apesar da primeira lei ter sido criada em 1970, progressos nos direitos de deficientes demoraram décadas. Antes da década de 90, de acordo com Stibbe (2004), deficientes eram invisíveis na televisão, assim como na sociedade. Eles apareciam às vezes em documentários e programas educacionais, mas jamais no horário nobre. A introdução de um personagem deficiente ocorreu em 1993 com o dorama Hitotsu Yane no Shita (Debaixo do mesmo teto), em que um personagem secundário era cadeirante. Mas, foi com Hoshi no Kinka (Moeda de ouro de estrelas), no qual a protagonista era uma enfermeira surda, que personagens deficientes (principalmente com deficiência auditiva) entraram na cultura popular. Sugimoto (2001, apud STIBBE, 2004) explica que doramas tiveram um impacto significativo na sociedade japonesa, introduzindo conceitos como bariafurī (barrier-free, ou seja, locais sem barreiras físicas) e causando um boom na procura de cursos de linguagens de sinais. 
Desde então, há cada vez mais séries de televisão que lidam com deficiências, inclusive em animações: seja de maneira fantástica (por meio de transmutação em Fullmetal Alchemist ou por contrato com demônios no clássico de Tezuka, Dororo) ou de maneira realista (a perda de um braço em uma explosão durante a Segunda Guerra Mundial, em Kono Sekai no Katasumi ni (Neste Canto do mundo), ou surdez como condição congênita em Koe no Katachi (A forma da voz). Em todos os exemplos citados, os portadores de deficiência são os heróis e as séries não têm medo de mostrar as dificuldades de seu dia-a-dia (O'REILLY, 2018).

Como os deficientes geralmente vivem em ambientes separados do restante da sociedade e são até mesmo escondidos por suas famílias, o único contato que a maioria dos japoneses têm com a condição de deficiência é por meio de sua representação fictícia (STIBBE, 2004). A mídia possui, assim, o papel de conscientizar a sociedade sobre a deficiência.

Essa invisibilidade dificulta até mesmo a obtenção de dados. Oficialmente, o Japão possui aproximadamente 7,8 milhões de portadores de deficiência, ou seja, $6 \%$ da população total (CABINET OFFICE, 2015). Esta quantidade é significativamente inferior à de outros países, por exemplo: o Reino Unido possui 27,2\% e a Finlândia, 32,2\% (MATSUI, 2009 , p.1). Há vários motivos para tal, como desejo por parte do indivíduo ou de familiares de esconder a condição, coleta de dados sem frequência e metodologia universal, além da ausência de um órgão responsável pela coleta. Até 1973, as coletas eram realizadas por meio de visitas à cada casa com questionários e entrevistas, mas foram abolidos por infringir o direito de privacidade. O novo método de coleta de dados ocorreu por meio de hospitais regionais, mas sem escala nacional (OKUNO, 1998; HEYER, 2000b; OI, 2009). Assim, os dados não necessariamente refletem a realidade do país.

O Japão reconhece oficialmente três tipos de identificação de deficiência: (i) deficiência física, (ii) deficiência intelectual e (iii) deficiência psicológica. No primeiro tipo, enquadram-se casos de comprometimento físico e deficiências visuais e auditivas. $\mathrm{O}$ segundo lida com atraso no aprendizado, limitações na comunicação, cuidado pessoal e habilidades sociais. E o terceiro com anormalidades de ordem psicológica, como esquizofrenia (SUZUKI, 2009; LO, 2012; MAIOR, 2016). O último levantamento realizado pelo governo foi em 2015, e constava que havia 3.937.000 deficientes físicos (31\%), 741 mil pessoas com deficiência intelectual (6\%) e 3.201.000 pessoas com deficiência psicológica (25\%) (CABINET OFFICE, 2015).

Para que um deficiente seja legalmente reconhecido como tal, ele deve portar um documento de identificação e um guia sobre deficiência, que lista o nome, tipo e severidade da condição. Não é obrigatório portar o documento, mas é o que possibilita receber benefícios econômicos ${ }^{4}$ e descontos em serviços sociais e médicos. Apenas indivíduos com este documento são contabilizados para cotas de deficiência em empresas.

4 Os benefícios econômicos incluem redução de impostos e isenção de outros, subsídios na compra de itens de ajuda mecânicos ou eletrônicos e descontos em transporte público. Adultos com condições graves que recebem um salário anual inferior a 3,4 milhões de ienes (o equivalente a 34 mil dólares) podem receber 26.800 ienes mensalmente. Famílias com crianças deficientes têm direito a 33 mil a 50 
A crítica de grupos ativistas é de que há um estigma e vergonha relacionados a este tipo de identificação (HEYER, 2000a).

No Japão, há um profundo estigma contra aqueles que são incapazes de trabalhar (ADAMS, 2016). O emprego é um símbolo-chave do status masculino e a incapacidade de poder exercer este papel invoca um sentimento de fracasso, principalmente para homens deficientes (TAKAGI, 2016). Mulheres deficientes, contudo, sofrem ainda mais vulnerabilidade. Para elas, a oportunidade de independência pode ser praticamente inexistente. Elas possuem menos probabilidade de se casarem e, no caso de mulheres que adquiriram uma deficiência após o casamento, há maior probabilidade de divórcio (GILL; CAROL, 1996 apud LEE, 1998).

Há um sentimento de vergonha em ter parentes "anormais", sendo considerado o dever da família (principalmente mulheres) não apenas cuidar, mas até mesmo escondê-los da sociedade (STEVENS, 2007 apud TAKAGI, 2016).

Este estigma pode ser notado com a tragédia de Sagamihara, o maior extermínio em massa no Japão desde a Segunda Guerra Mundial, que ocorreu em 2016. O ataque ocorreu na clínica Tsukui Yamayuri En, em Kanagawa, especializada em cuidar de pacientes com deficiências mentais, onde 19 pessoas foram mortas e 26 feridas. $\mathrm{O}$ autor do massacre foi um ex-funcionário, Satoshi Uematsu, que defendeu o ato "em nome do Japão e da paz mundial" (HERNON, 2017, p.1), alegando que deficientes deveriam desaparecer.

Diferentemente do que ocorre no Ocidente, a polícia não revelou à mídia a identidade das vítimas. Esta decisão tinha como objetivo focar na proteção das famílias das vítimas. Esta ação intensificou o debate em relação ao preconceito existente contra pessoas com deficiência. De acordo com Suzanne Kamata (HERNON, 2017, p.1), "Não mostrar seus nomes e rostos é como negar a sua humanidade. O incidente não recebeu o mesmo nível de atenção como outras tragédias, não houve hashtags no Twitter e o assunto morreu rapidamente". A falta de informação sobre as vítimas dificulta a criação de empatia em relação a elas (ADAMS, 2016; HA; SIEG, 2016; HERNON, 2017).

Outra questão que vem sido discutida é a Lei de Proteção Eugênica de 1948, que autorizava legalmente a esterilização, com ou sem consenso, de portadores de deficiência e doenças mentais hereditárias ${ }^{5}$ para "prevenir o nascimento de descendentes inferiores (...), proteger a vida e saúde da mãe (...), melhorar a qualidade da nação" (HURST, 2018, p.2). Mesmo os indivíduos que consentiram o procedimento muitas vezes o fizeram sob ameaça de médicos e familiares. A lei perdurou por quase cinco décadas, sendo revogada apenas em 1996 (HURST, 2018; McCURRY, 2018).

Esta lei gera repercussões até hoje, pois entre 1948 e 1996 aproximadamente 25 mil pessoas foram esterilizadas, incluindo 16.500 pessoas que foram submetidas à

mil ienes por mês dependendo do grau da deficiência, e mais 14 mil ienes se as crianças requererem cuidados constantes (HEYER, 2000b; PENKETH, 2015).

5 A lei passou a incluir condições não-hereditárias após revisões na mesma década. 
esterilização forçada. Desses casos, $70 \%$ envolveram mulheres, sendo que as pacientes mais jovens tinham apenas 10 anos (HURST, 2018).

Em 2016, o Comitê da ONU de Eliminação da Discriminação contra Mulheres apelou ao governo japonês para adotar "medidas com objetivo de providenciar acesso a remédios legalizados, compensação e serviços de reabilitação a todas as vítimas de esterilização forçada" (McCURRY, 2018, p.2). Mas, foi apenas a partir de 2018 que discussões sobre compensações se iniciaram. Este processo é liderado pelas vítimas, alegando que a lei é anticonstitucional e que infringiu seus direitos de autonomia corporal. As vítimas buscam uma compensação de 11 milhões de ienes, o equivalente a quase 100 mil dólares (ASAHI SHIMBUN, 2018a). O governo demonstrou interesse em conversar com as vítimas, mas alegou que não tinha planos de providenciar compensações para todas, por não ter responsabilidade legal em realizá-lo (McCURRY, 2018; ASAHI SHIMBUN, 2018a).

Outros países que realizaram planos similares, como a Alemanha e Suécia, se desculparam publicamente às vítimas e lhes providenciaram compensação financeira, enquanto o governo japonês não compensou nenhuma vítima até hoje (McCURRY, 2018; ASAHI SHIMBUN, 2018a). Recentemente, houve sinais de possível evolução. Há planos de realização de missões de averiguação em conjunto com autoridades locais, o que pode ser um possível precedente para desculpas públicas (HURST, 2018).

Para Hurst (2018), apesar de esterilizações forçadas terem sido abolidas em 1996, as atitudes discriminatórias que existiam por trás da lei ainda estão presentes na atual sociedade japonesa.

O Japão possui hoje um Estado de bem-estar social sofisticado, com boas instituições de reabilitação separadas do restante da sociedade, mas às custas da igualdade e integração. E, apesar das melhorias na infraestrutura com a criação de áreas "sem barreiras" durante as últimas décadas, portadores de deficiência são sistematicamente tratados como invisíveis pela sociedade (OTAKE, 2006).

Outra tradição ainda comum é a institucionalização de deficientes. As crianças portadoras estudam em escolas especiais (yôgo gakkô), cada qual dedicada exclusivamente a específicos tipos de deficiências e, ao graduar, ou elas vivem em centros de reabilitação públicos e privados ${ }^{6}$ ou trabalham em armazéns afastados (jusan shisetsu) ou em empresas de bem-estar social (fukushi kôjô). Em suma, a condição de isolamento perdura durante suas vidas (ADAMS, 2016).

Em 2006, houve uma reforma educacional, o Ato Base de Educação (Kyôiku kihon-hô) (VISLIE, 2003 apud MITHOUT, 2016). Esta reforma teve como princípio a ideia de "educação para todos", na qual escolas teriam a obrigação de atender às demandas de todas as crianças, independentemente de particularidades em habilidades, conhecimento da língua japonesa e origem étnica, social ou familiar. De acordo com Mithout (2016),

6 Esses centros não oferecem proteção dos direitos do trabalhador e pagam um salário inferior a 30.000 ienes por mês, o que impossibilita um deficiente viver independentemente (OTAKE, 2006). 
isso é um alívio financeiro, pois as escolas especiais são caras, devido à sua baixa proporção de aluno-professor (de até 6 alunos por professor).

Contudo, o sucesso dessa reforma depende do tipo de deficiência. Crianças com deficiências visuais e auditivas continuaram encontrando grandes obstáculos para realizar os vestibulares, enquanto deficientes físicos tiveram dificuldade de locomoção nas escolas. As crianças autistas e com deficiência de desenvolvimento obtiveram o maior sucesso de adequação (MITHOUT, 2016). O aumento na quantidade de estudantes com "deficiências invisíveis" em escolas convencionais pode aumentar o reconhecimento social delas, mas não é uma solução, pois limita o acesso e interação para crianças com outros tipos de deficiências (DISABLED WORLD, 2010; HEYER, 2000a).

As atitudes de professores, administradores de escolas, outras crianças e até mesmo de familiares também são barreiras à sua inclusão no ensino convencional (WHO, 2011). A segregação na infância torna-se um dos maiores obstáculos para seu futuro. As crianças com deficiência crescem em uma bolha, tornando-se despreparadas para lidar com o mundo real e, na maioria dos casos, limitando permanentemente suas chances de ingressar na sociedade. Quando chegam à idade adulta, portadores de deficiência tendem a viver com os pais, em instituições ou em lares coletivos, o que dificulta ou mesmo impossibilita seu ajustamento às normas sociais e às longas horas de trabalho (KUDO, 2010).

O sistema de cotas introduzido com a Lei de Promoção de Emprego para Pessoas com Deficiências Físicas ${ }^{7}$ em 1960 visa amenizar a situação, estabelecendo a obrigação de reservar, no mínimo, $2,2 \%$ do total das vagas no setor privado ${ }^{8}$ e de $2,5 \%$ no público (INAGAKI, 2018). No caso de descumprimento, há uma multa de 50 mil ienes (454 dólares). A lei também requer que o empregador crie "acomodações razoáveis" para que o trabalhador não enfrente dificuldades desmerecidas (SIM, 2018; ASAHI SHIMBUN, 2018b; LO, 2012). Contudo, as cotas nunca foram preenchidas. O aumento na empregabilidade só ocorreu após a publicação dos nomes de empresas que não preencheram a cota. A mídia e a pressão da opinião pública alteraram seu comportamento. Para Mori e Sakamoto (2018), as normas sociais aparentam ser um dos principais fatores no incentivo da empregabilidade de portadores de deficiência, mesmo quando não há pagamento de multas.

A mídia, a sociedade e o setor privado criticaram severamente a postura governamental após ser revelado que diversos ministérios declararam uma quantidade de empregados deficientes maior do que o número real. O sistema de cotas japonês é um dos mais complexos do mundo, mas a confiabilidade no sistema está abalada após saber que o governo, órgão que deveria ser um exemplo para a sociedade, além de responsável

7 Inicialmente, a lei apenas incluía deficientes físicos. Em 1987, portadores de deficiência mental foram incluídos, apesar de sua contratação ainda não ser obrigatória.

8 Para empresas com, no mínimo, 45 trabalhadores (INAGAKI, 2018). 
por regular e punir o setor privado, manipulou dados para aparentar ser inclusivo (SHIMAMOTO e MURAKOSHI, 2018; SIRIPALA, 2018; SIM, 2018).

A inexistência de uma instituição fiscalizadora de escala nacional independente do governo dificulta tanto a coleta e verificação de dados quanto a mobilização social. O caráter de soft law ${ }^{9}$ da maioria das leis japonesas dificulta sua implementação devido à falta de caráter punitivo. Há uma tradição de providenciar apenas o "dever de comprometimento" 10 , devido ao temor de que o impacto de leis obrigatórias venha a ameaçar a harmonia da sociedade japonesa (PECKITT, 2016; NAKAGAWA, 2010). Kristof (1996) argumenta que esta harmonia é adquirida ao excluir todos aqueles que não se encaixam. Essa exclusão significa que as pessoas com deficiência são frequentemente desencorajadas de se casarem, trabalharem ou mesmo saírem para fins de lazer, como ir para cinemas e restaurantes. Ou seja, de viverem em sociedade.

\section{CONCLUSÃO}

Uma transformação está ocorrendo no Japão com o aumento da conscientização de direitos e por meio da criação e implementação de leis como instrumento de mudança social (HEYER, 2000b). Contudo, ainda existem estigmas baseados em concepções preconceituosas, assim como falta de interesse e pro-atividade por parte do governo, restando a grupos ativistas e às vítimas de discriminação demandarem publicamente por mudanças.

As Paralimpíadas oferecem não só uma oportunidade para trazer à realidade um tema que para muitos ainda é abstrato ou desconhecido, mas também uma maneira de testar a infraestrutura do país receptor. Na China e na Rússia, após os Jogos, houve maior conscientização e discussão sobre as condições de portadores de deficiência. 200 cidades russas usam hoje Sochi como modelo. O mesmo ocorreu com Barcelona em 1992, que hoje é uma das cidades mais acessíveis da Europa (CRAVEN, 2016).

Craven (2016) enfatiza a importância do papel das Paralimpíadas em empoderar indivíduos por meio da inclusão social. Para Blauwet (2005), não há dúvida de que a paixão demonstrada no esforço e na capacidade de atletas paralímpicos se traduzirá na sensibilização e defesa de empreendimentos em outras áreas, como no estudo e trabalho. Contudo, Marshall (1998, apud STIBBE, 2002) aponta que há uma diferença no tratamento entre deficientes que superam suas barreiras com esportes e deficientes "comuns". As narrativas de atletas focam na capacidade do indivíduo superar sua tragédia individual. A sociedade aparenta não ter papel algum. Van Der Veere (2020) aponta que isso é problemático, pois essas histórias individualizam a deficiência, em vez de lidar com os problemas sociais que portadores enfrentam no seu dia a dia. Torna-se importante a "apreciação de pessoas com todos os tipos de

9 Normas não juridicamente vinculantes.

10 "Duty to endeavor" no original (NAKAGAWA, 2010). 
deficiência, independentemente se elas conseguem ou não esquiar montanha abaixo" (MARSHALL, 1998, apud STIBBE, 2002, p.15).

Portanto, mesmo que a sociedade veja paralímpicos de maneira positiva, não necessariamente isso se aplicará para todos os tipos de portadores de deficiência. As Paralimpíadas podem ajudar no processo, mas elas são apenas uma parte. A sociedade, o governo e o setor privado não devem esquecer-se da importância de seus papéis.

\section{BIBLIOGRAFIA}

ADAMS, Rachel. Why has Japan's massacre of disabled people gone unnoticed? The Independent, 2016. Disponível em: https://www.independent.co.uk/voices/japan-disability-rights-massacre-tsukui-yamayuriena-gone-unnoticed-a7217661.html Acesso em 2 set. 2018.

ASAHI SHIMBUN. Defend eugenics program with the Constitution, court tells state. Tokyo, 2018a. Disponível em: http://www.asahi.com/ajw/articles/AJ201806140059.html Acesso em 8 out. 2018.

. Ministries may have skirted disability hiring for many years. Tokyo, 2018b. Disponível em: http://www.asahi.com/ajw/articles/AJ201808180031.html Acesso em 19 mar. 2019.

BLAUWET, Cheri. Promoting the Health and Human Rights of Individuals with a Disability through the Paralympic Movement. Bonn: International Paralympic Committee, 2005. Disponível em: https://www.sportanddev.org/sites/default/files/downloads/62_the_paralympics__promoting_health_and_human_rights_through_sport.pdf Acesso em 13 ago. 2020.

BRASOR, Philip. Accommodating disabilities, but only within reason. Tokyo: Japan Times, 2016. Disponível em: https://www.japantimes.co.jp/news/2016/04/16/national/media-national/accommodating-disabilities-within-reason/ Acesso em 4 set. 2018.

CABINET OFFICE. Annual Report on Government Measures for Persons with Disabilities (Summary). 2015. Disponível em: https://www8.cao.go.jp/shougai/english/annualreport/2015/index-pdf.html Acesso em 11 ago. 2020.

CABINET OFFICE. The Basic Law for Persons with Disabilities. 2011. Disponível em: https:// www8.cao.go.jp/shougai/english/law/no84.html Acesso em 11 ago. 2020.

CARVALHO, Lucio; ALMEIDA, Patricia. Direitos Humanos e Pessoas com Deficiência: da exclusão à inclusão. Da proteção à promoção. Revista Internacional de Direito e Cidadania, n.12, 2012. Disponível em: https://www.inclusive.org.br/arquivos/30688 Acesso em 22 ago. 2020.

CRAVEN, Philip. The Paralympic Games and the Promotion of the Rights of Persons with Disabilities. UN Chronicle, v.LIII, n.2, 2016. Disponível em: https://unchronicle.un.org/article/ paralympic-games-and-promotion-rights-persons-disabilities Acesso em 12 ago. 2020.

DISABLED WORLD. Disability in Japan. 2010. Disponível em: https://www.disabled-world. com/news/asia/japan.php Acesso em out. 2018. 
FRENCH, Sally. Disability, impairment, or something in between?. In: SWAIN, John; FINKELSTEIN, Vic; FRENCH, Sally; OLIVER, Mike (eds). Disabling Barriers - Enabling Environments. Londres: Sage, 1993, p.17-25.

HA, Kwiyeon; SIEG, Linda. Japan confronts disability stigma after silence over murder victims' names. Reuters, 2016. Disponível em: https:/www.reuters.com/article/us-japan-disabled-idUSKCN11M0AM Acesso em 15 out. 2018.

HASEGAWA, Tamako. Reasonable Accommodation for Persons with Disabilities in Japan. Japan Labor Review, v12, n.1, 2015.

HERNON, Matthew. Why is Japan still biased against people with disabilities. Tokyo Weekender, 2017. Disponível em: https://www.tokyoweekender.com/2017/02/why-is-japan-still-biased-against-people-with-disabilities/ Acesso em 22 set. 2018.

HEYER, Katharina. Between equality and difference: the politics of disability in Japan. Contemporary Japan, v.1, p.105-133, 2000.

HEYER, Katharina. From special needs to equal rights: Japanese disability law. Asian-Pacific Law \& Policy Journal, 2000. Disponível em: http://blog.hawaii.edu/aplpj/files/2011/11/ APLPJ_01.1_heyer.pdf Acesso em 16 jun. 2020.

HURST, Daniel. 'They stole my life away': women forcibly sterilised by Japan speak out. The Guardian, 2018. Disponível em: https://www.theguardian.com/world/2018/apr/04/they-stole-my-life-away-women-forcibly-sterilised-by-japan-speak-out Acesso em 27 fev. 2020.

INAGAKI, Kana. Japan companies in 'hiring war' to hit disability targets. Financial Times, 2018. Disponível em: https://www.ft.com/content/44cac7dc-3be5-11e8-bcc8-cebcb81f1f90 Acesso em 19 jan. 2020.

ITAYAMA, Kenji. Outlook on Changes in the Social Welfare System in Japan: Key Points of Its Basic Structural Reform. Asia and Pacific Journal on Disability, v.2, 1999. Disponível em: https://www.dinf.ne.jp/doc/english/asia/resource/z00ap/004/z00ap00411.html Acesso em 15 mar. 2020.

KATSUI, Hisayo. Mainstreaming Disability Issues in Japanese and Finnish Development Policies and Practices. Helsinki University: Institute of Development Studies, 2008. Disponível em: https://www.sylff.org/wp-content/uploads/2008/12/mainstreaming_katsui_hisayo.pdf Acesso em 21 abr. 2020.

KRISTOF, Nicholas. Outcast Status Worsens Pain of Japan's Disabled. The New York Times, 1996. Disponível em: https://www.nytimes.com/1996/04/07/world/outcast-status-worsens-pain-of-japan-s-disabled.html Acesso em 14 ago. 2020.

KRUG, Etienne. International paralympic symposium on disability rights. World Health Organization, 17 September 2004. Disponível em: https:/www.who.int/violence_injury_prevention/media/17_09_2004_speech/en/ Acesso em 22 ago. 2020.

KUDO, Tokai. Japan's Employment Rate of Persons with Disabilities and Outcome of Employment Quota System. Japan Labor Review, v.7, n.2, 2010. Disponível em: https://www.jil. go.jp/english/JLR/documents/2010/JLR26_kudo.pdf Acesso em 14 ago. 2020. 
LEE, Jennifer; WHITE, Shirley. Economic Sufficiency of Women with Disability. Asia and Pacific Journal on Disability, v.3, 1998. Disponível em: https://www.dinf.ne.jp/doc/english/ asia/resource/z00ap/003/z00ap00305.html Acesso em 20 jul. 2020.

LO, Vai Io. Promotion of the employment of persons with disabilities in Japan, the United States, and China: carrot, stick or both? Arizona Journal of International \& Comparative Law, v.29, n.3, p.557-602, 2012.

MACKAY, Duncan. Major initiative launched to help Japanese athletes prepare for Tokyo 2020 Paralympics. Inside the games, 2015. Disponível em: https:/www.insidethegames. biz/articles/1028374/major-initiative-launched-to-help-japanese-athletes-prepare-for-tokyo-2020-paralympics Acesso em 15 ago. 2020.

MAIOR, Izabel. História, conceitos e tipos de deficiência. Gov. SP, 2016. Disponível em: http:// violenciaedeficiencia.sedpcd.sp.gov.br/pdf/textosApoio/Texto1.pdf Acesso em 10 fev. 2020.

MATSUI, Akihiko. Disability and Economy in Japan. University of Tokyo, 2009. Disponível em: http:/www.rease.e.u-tokyo.ac.jp/read/en/archive/dp/f09/f0911.pdf Acesso em 17 mai. 2020.

McCURRY, Justin. Japanese woman sues government over forced sterilisation. The Guardian, 2018. Disponível em: https://www.theguardian.com/world/2018/jan/30/japanese-woman-sues-government-forced-sterilisation Acesso em 22 jun. 2020.

MITHOUT, Anne-Lise. Children with disabilities in the Japanese school system: a path toward social integration? Contemporary Japan, v.28, p.165-184, 2016.

MORI, Yuko; SAKAMOTO, Norihito. Economic consequences of employment quota system for disabled people: Evidence from a regression discontinuity design in Japan. Journal of The Japanese and International Economies, v.28, 2018. Disponível em: https:/www. sciencedirect.com/science/article/pii/S0889158317300035 Acesso em 11 ago. 2020.

NAGASE, Osamu. Challenges of the Harmonization and Ratification of Convention on the Rights of Persons with Disabilities by Japan. Journal of Policy and Practice in Intellectual Disabilities, v.10, n2, p.93-95, 2013.

NAKAGAWA, Jun; BLANCK, Peter. Future of Disability Law in Japan: Employment and Accommodation. Loyola Marymount University and Loyola Law School, 2010. Disponível em: https://digitalcommons.lmu.edu/cgi/viewcontent.cgi?article=1662\& context=ilr Acesso em 14 abr. 2020.

OI, Tatsuo. Statistics on disabled People in Japan. Aino Journal, v.8, p.3-10, 2009.

OKUNO, Eiko. Disability Statistics in Japan. Asia and Pacific Journal on Disability, v.3, 1998. Disponível em: https:/www.dinf.ne.jp/doc/english/asia/resource/z00ap/003/z00ap00309. html Acesso em 16 jun. 2020.

O’REILLY, Sean. “Disarmed”: Disability, Trauma, and Emasculation in Contemporary Japanese Cinema. Arts, v.7, art.10, 2018. 
OTAKE, Tomoko. Is 'disability' still a dirty word in Japan? Japan Times, The. 2006. Disponível em: https://www.japantimes.co.jp/life/2006/08/27/to-be-sorted/is-disability-still-a-dirty-word-in-japan/\#.UyFLy-eSyzc Acesso em 10 nov. 2019.

PECKITT, Michael. Disability in Japan - A year in review: 2016. Accessible Japan, 2016. Disponível em: https:/www.accessible-japan.com/disability-japan-year-in-review-2016/ Acesso em 14 abr. 2020.

PENKETH, Anne; CONNOLLY, Kate; KIRCHGAESSNER, Stephanie, McDONALD, Henry; McCURRY, Justin; CROUCH, David; WALKER, Shaun; SMITH, David; O'HARA, Mary e BAWDEN, Anna. Which are the best countries in the world to live in if you are unemployed or disabled? The Guardian, 2015. Disponível em: https://www.theguardian. com/politics/2015/apr/15/which-best-countries-live-unemployed-disabled-benefits Acesso em 21 abr. 2020.

RAJNES, David. Permanent Disability Social Insurance Programs in Japan. Social Security Bulletin, v.70, n.1, 2010.

SHIRASAWA, Mayumi. The Long Road to Disability Rights in Japan. Nippon.com, 2014. Disponível em: https://www.nippon.com/en/currents/d00133/the-long-road-to-disability-rights-in-japan.html Acesso em 10 mar. 2020.

SIM, Walter. Inflated number of staff with disabilities at Japan ministries. The Straits Times, 2018. Disponível em: https:/www.straitstimes.com/asia/east-asia/inflated-number-of-staff-with-disabilities-at-japan-ministries Acesso em 19 maio 2020.

SIRIPALA, Thinsaka. Japan's Government Investigated for Cheating Disability Hiring Quotas. The Diplomat, 2018. Disponível em: https://thediplomat.com/2018/08/japans-government-investigated-for-cheating-disability-hiring-quotas/ Acesso em 17 jul. 2020

STIBBE, Arran. Attitudes to Women with Disabilities in Japan: The Influence of Television Drama. Asia and Pacific Journal on Disability, v.5, 2002. Disponível em: https:/www. dinf.ne.jp/doc/english/asia/resource/z00ap/vol5no2/attitudes.html Acesso em 9 mar. 2019.

STIBBE, Arran. Disability, gender and power in Japanese television drama. Japan Forum, v.16, p.21-36. Disponível em: https://www.tandfonline.com/doi/abs/10.1080/0955580032000189311 Acesso em 20 mar. 2020.

SUGIMOTO, Kiyoe. Byūtifuru raifu wa bariafurī de. Sanseiken Forum 49, 2001. Disponível em: www.sanseiken.com/forum/40/49-otoko.html Acessado por Stibbe (2004) em 2002.

SUZUKI, Tsunehiko. Disability Evaluation in Japan. The Korean Academy of Medical Sciences, v. 24, p. 227-231, 2009.

TACHIBANA, Toshiaki; WATANABE, Kanji. Attitudes of Japanese Adults toward Persons with Intellectual Disability: Relationship between Attitudes and Demographic Variables. Education and Training in Developmental Disabilities, v.39, p.109-126, 2004.

TAGAKI, Masakuni. Narratives of Ambivalent Meanings of Acquired Physical Disability in Japan. SAGE Open, 2016. Disponível em: https://journals.sagepub.com/ doi/10.1177/2158244016666310 Acesso em 24 jun. 2020. 
TAKAHASHI, Koji. The Future of the Japanese-style Employment System: Continued Long-term Employment and the Challenges It Faces. Japan Labor Issues. 4-5. v.2, n.6, 2018.

VAN DER VEERE, Anoma. The Tokyo Paralympic Superhero: Manga and Narratives of Disability in Japan. The Asia-Pacific Journal, v.18, n.5, 2020. Disponível em: https://apjjf. org/-Anoma-van-der-Veere/5373/article.pdf Acesso em 16 ago. 2020.

WORLD HEALTH ORGANIZATION. World Report on Disability: Summary. Geneva, p.8-10, 2011. Disponível em: https://apps.who.int/iris/handle/10665/44575 Acesso em 14 mar. 2020. 